\title{
Routine cholangiography during rigid-hybrid transvaginal natural orifice transluminal endoscopic cholecystectomy
}

\author{
Önder Ögredici · Georg R. Linke · Sebastian Lamm • \\ Rachel Rosenthal · Andreas Zerz $\cdot$ Daniel C. Steinemann
}

Received: 6 May 2013/Accepted: 4 September 2013/Published online: 19 October 2013

(C) Springer Science+Business Media New York 2013

\begin{abstract}
Background Transvaginal rigid-hybrid transluminal endoscopic cholecystectomy (tvCCE) has become a routine procedure in some laparoscopic departments in recent years. Although intraoperative cholangiography is an important adjunct to cholecystectomy, its feasibility and safety in tvCCE have not been demonstrated to date.

Methods Patients undergoing tvCCE between April and October 2012 were included in this study. An intraoperative cholangiogram was obtained routinely for all the patients. Patient characteristics, operation data, feasibility, and duration of the cholangiography as well as the postoperative course were recorded prospectively.

Results For 32 (97\%) of the 33 patients enrolled in this study, intraoperative cholangiography could be performed successfully. The median duration of cholangiography was
\end{abstract}

Presented at the 21st International Congress of the European Association for Endoscopic Surgery (EAES), Vienna, Austria, 19-22 June 2013.

Ö. Ögredici · S. Lamm · A. Zerz · D. C. Steinemann $(\bowtie)$

Department of Surgery, Kantonsspital Baselland,

CH-4101 Bruderholz, Switzerland

e-mail: daniel.steinemann@gmx.ch

Ö. Ögredici

Department of Surgery, Kantonsspital St. Gallen,

Rorschacherstrasse 110, 9007 St. Gallen, Switzerland

G. R. Linke

Department of General, Visceral and Transplantation Surgery, University Hospital Heidelberg, Im Neuenheimer Feld 110, 69120 Heidelberg, Germany

R. Rosenthal

Department of Visceral Surgery, University Hospital Basel, Spitalstrasse 21, 4056 Basel, Switzerland
6 min (interquartile range, 4-7 min). Common bile duct stones were detected in three patients $(10 \%)$. Laparoscopic bile duct revision with the aid of one additional port was successful in two of these patients. One patient needed postoperative endoscopic retrograde cholangiopancreatography due to the impossibility of extracting an impacted prepapillary concrement. One operation was converted to a four-port laparoscopic cholecystectomy. One additional port was used in 11 patients $(33 \%)$ and two additional ports in three patients $(9 \%)$. Three intraoperative minor complications $(9 \%)$ and one postoperative minor complication (3\%) occurred.

Conclusions Intraoperative cholangiography during tvCCE is feasible, safe, and easy to perform. The need for intraoperative cholangiography no longer represents a contraindication for tvCCE.

Keywords Transvaginal · Hybrid .

Cholecystectomy · Intraoperative cholangiography ·

NOTES

Since the first description of transvaginal rigid-hybrid transluminal endoscopic cholecystectomy (tvCCE) in 2007 [1], this technique has evolved and been introduced into many clinics. In some specialized departments, transvaginal access for elective cholecystectomy in women has become a standard procedure. The potential advantages of this technique are reduced scars [1-7], reduced risk for trocar hernia, and above all, reduced postoperative pain $[4$, 7-9].

Overall, findings have shown tvCCE to be feasible and safe [1-6]. Moreover, gynecologic complications such as dyspareunia [6] and infectious complications [10] are not increased during long-term follow-up evaluation. Yet, no 
randomized controlled trials have examined the influence of this new technique on the rate of biliary tract injuries, which is the main safety issue in cholecystectomy [11].

With the introduction of laparoscopic cholecystectomy in the 1990s, the incidence of biliary tract injuries increased rapidly [12] but then fell as the technique became standardized and routine. The incidence of biliary injury in laparoscopic cholecystectomy currently is as high as $0.3 \%$ [13]. With the introduction of tvCCE, the risk of an increased biliary tract injury rate may emerge again during the period of the learning curve.

Intraoperative cholangiography (IOC), an important adjunct of laparoscopic cholecystectomy, is used to clarify the anatomy of the biliary system to rule out biliary injury if suspected or if any doubt exists [14] and to search for choledocholithiasis. It is unclear whether routine IOC is generally useful or whether it should be used only selectively [15-18]. However, there is a consensus that IOC plays an important role as an adjunct to cholecystectomy.

Only in reports of three cases has the feasibility of IOC during tvCCE been demonstrated [5]. Currently, prospective series monitoring IOC in routine tvCCE are lacking. This study aimed to evaluate the feasibility and safety of routine IOC during tvCCE.

\section{Materials and methods}

From April to October 2012, tvCCE was offered to all female patients with symptomatic cholecystolithiasis and in selected cases to women with acute cholecystitis. Patients choosing transvaginal cholecystectomy were included in this prospective cohort study and underwent IOC during tvCCE.

The inclusion criteria specified any female patient older than 18 years with symptomatic gallstone disease. Patients provided written informed consent before surgery. The exclusion criteria ruled out patients with missing informed consent, pregnancy, vaginal atresia, florid vaginal infection, gynecologic neoplasia, or allergy to contrast media. The study was approved by the local ethics committee of cantons Basel-Town and Basel-Country, Switzerland, and registered at clinicaltrials.gov (NCT01583348).

A preoperative gynecologic examination was performed by a consultant gynecologist. At the admission date, a pregnancy test was performed. Patient age, body mass index (BMI), leading symptoms, history of abdominal surgery, and the results of sonography, magnetic resonance cholangiopancreatography (MRCP), and endoscopic retrograde cholangiopancreatography (ERCP) were obtained.

The operating data were recorded including operation time, duration of cholangiography (from clipping of the cystic duct to dissection of the cystic duct after cholangiography), number of trocars used, and type of cholangiography catheter used. The postoperative data included the length of the postoperative hospital stay and the gynecologic examination (bimanual palpation; inspection of the vagina, cervix, and posterior fornix; bacteriology test of the cervix) results 2 weeks after surgery.

The postoperative course was assessed in the outpatient department 6 weeks postoperatively. Complications were classified according to the Clavien-Dindo categorization [19].

\section{Surgical technique}

The tvCCE procedure is performed much the same as described previously [4]. The patient undergoes surgery in the lithotomy position on an x-ray-suitable operating table (Alphamaquet; Maquet AG, Gossau, Switzerland). The operating surgeon stands on the patient's left side, and the assistant stands between the legs (Fig. 1).

A single shot of antibiotic consisting of cefazolin is administered. The abdomen and the vagina are prepared (Octenisept is used for the vagina and Octeniderm for the skin; Schülke \& Mayr GmbH, Norderstedt, Germany). A $12-\mathrm{mmHg}$ carbon dioxide $\left(\mathrm{CO}_{2}\right)$ pneumoperitoneum is established using a Veress needle placed through the umbilicus, and a 5-mm trocar (Versastep; Covidien, Norwalk, CT, USA) is inserted through the umbilicus. A 5-mm optic then is introduced, and the patient is placed in Trendelenburg position.

At this point, after the urinary bladder has been catheterized, an intrauterine manipulator (ClearView; Clinical Innovations, Murray, UT, USA) is used to expose the posterior fornix. Under laparoscopic guidance, a 5-mm vaginal V-port (A.M.I. GmbH, Feldkirch, Austria) is inserted through the posterior fornix initially, followed by a 12-mm vaginal $\mathrm{V}$-port.

A $10-\mathrm{mm} 45^{\circ}$ endoscope (Olympus, Tokyo, Japan) and an extra-long curved grasper are placed through the transvaginal ports. The camera is switched to the transvaginal endoscope. The patient is repositioned in antiTrendelenburg position. The infundibulum of the gallbladder then is grasped and pulled in a craniolateral direction according to a three-port cholecystectomy technique [20]. If Calot's triangle cannot be exposed with this single-grasper retraction, enabling safe dissection, further abdominal trocars are inserted.

Dissection instruments are introduced through the umbilical port (Fig. 2A). According to the principle of critical view of safety [21], the peritoneum is opened wide in the medial and lateral aspects of the infundibulum. A window is developed between the gallbladder and the liver, and the cystic artery is released from the gallbladder wall. A critical safety triangle is prepared between the 
Fig. 1 Operating room setup

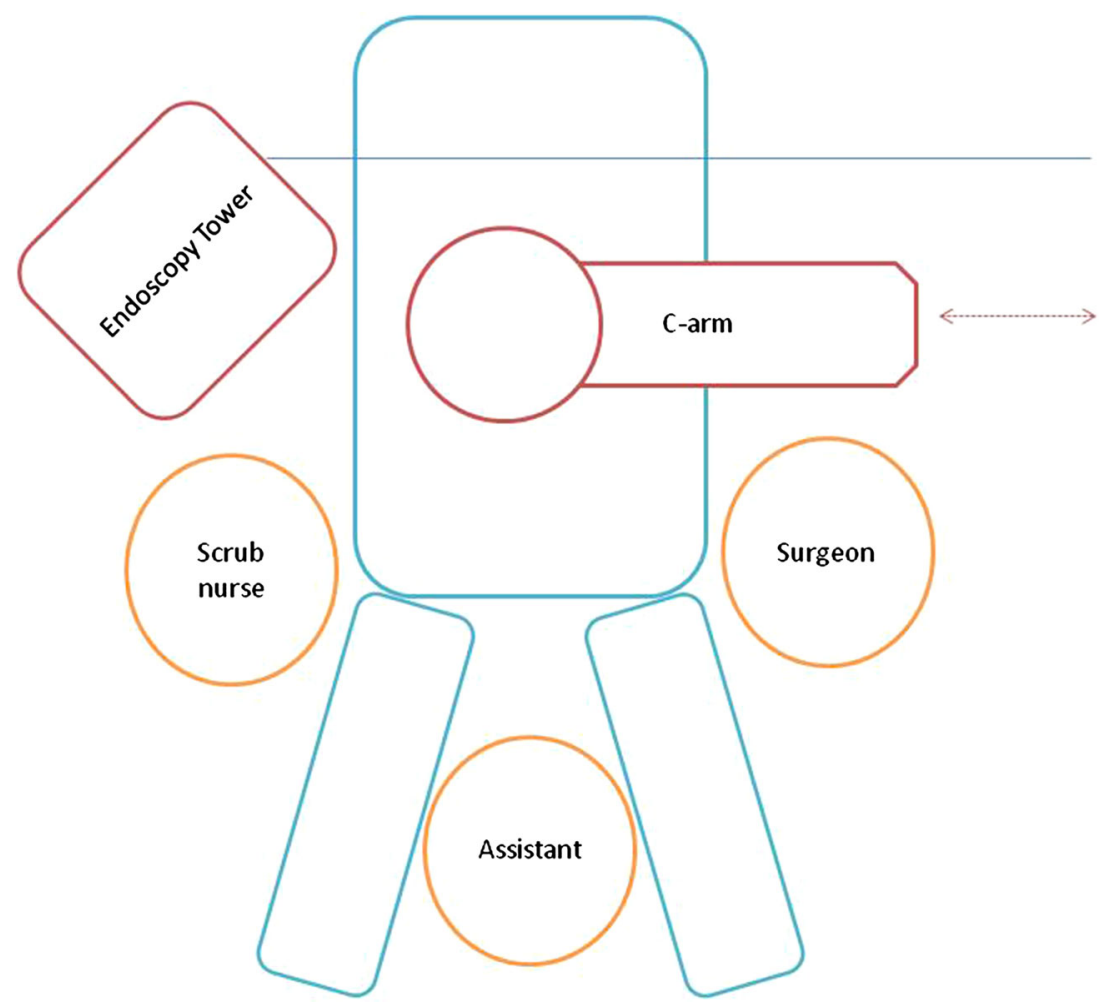

A

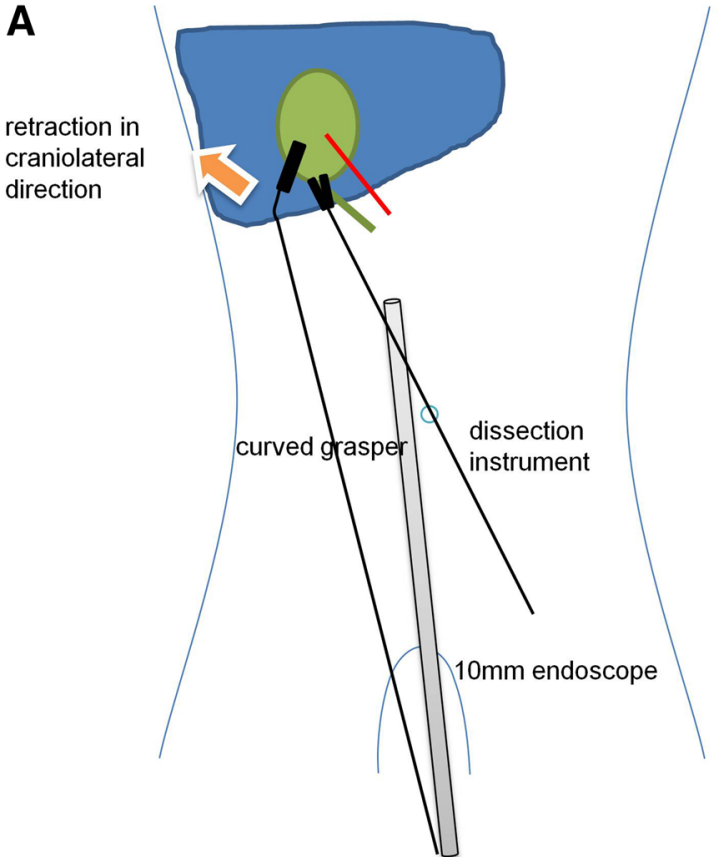

B

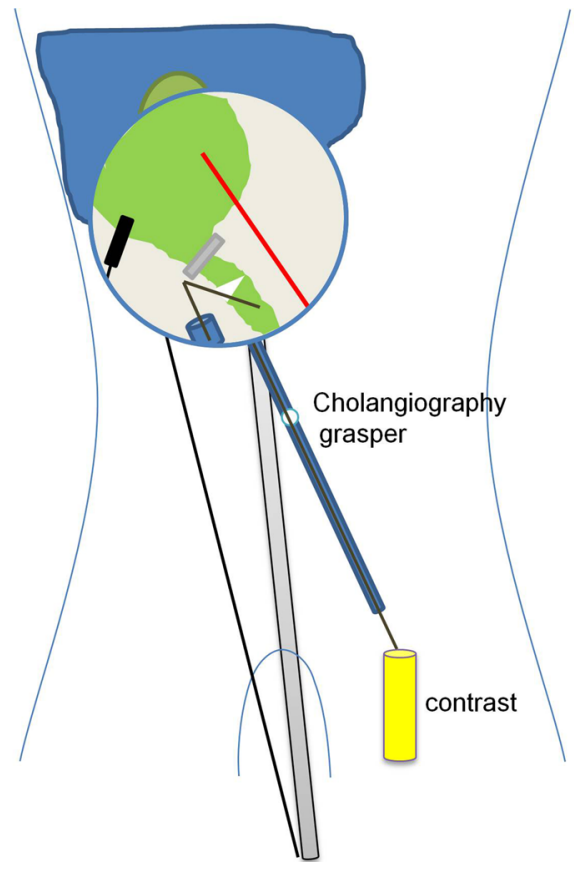

Fig. 2 Schematic view of the surgical procedure. A Retraction and dissection of Calot's triangle. B Insertion of cholangiography catheter

gallbladder wall, with the cystic duct inferiorly on the right and the cystic artery on the left [22].

After safe identification of both the cystic duct and the cystic artery, a 5-mm clip (Ligamax 5-mm Endoclips Applier; Ethicon, Cincinnati, OH, USA) is placed distally on the cystic duct. The cystic duct is incised using scissors proximal to the clip, and a cholangiography grasper (Karl Storz GmbH \& KG, Tuttlingen, Germany) is introduced through the umbilical port. A cholangiography catheter (Coeliodrain, 5 Fr, $35 \mathrm{~cm}$; Coloplast A/S, Humlebæk, Denmark, or Argyle polyurethane umbilical vessel catheter, 5 Fr, 38.1 cm; Covidien plc, Dublin, Ireland) is placed 
through the cholangiography grasper. The tip of the catheter is bent in the direction of the cystic duct. The duct then is intubated (Fig. 2B) and occluded with the help of the cholangiography grasper.

A fluoroscopic image intensifier C-arm (Pulsera, 12; Philips N.V., Amsterdam, Netherlands) is positioned from the left side just cranial to the operating surgeon. Contrast medium (Telebrix; Gastro, Guerbet Group, Villepinte, France) is injected, and a cholangiogram is obtained.

After extraction of the cholangiography catheter, the cystic duct is proximally clipped twice and cut. The cystic artery is clipped and divided, and a retrograde cholecystectomy is performed using transumbilically inserted shears. The gallbladder is placed in a $5-\mathrm{mm}$ endobag (TissueBag Premium; A.M.I. GmbH, Feldkirch, Austria) introduced through the umbilical port.

Again, the camera is switched to the umbilicus, and the curved grasper is removed together with the 5-mm vaginal port. The 10-mm transvaginal optic is removed, and a straight grasper is introduced through the remaining vaginal port. The cord of the closed endobag is grasped, and the gallbladder is removed through the colpotomy after removal of the remaining 12-mm V-port.

Finally, the colpotomy is sutured with absorbable thread. The pneumoperitoneum is deflated, and the umbilical Versastep port is removed.

Data collection and statistical analysis

Data were collected in a study database using Excel, version 12.0, 2007 (Microsoft Switzerland, Wallisellen, Switzerland). Descriptive statistical analysis was performed using GraphPad Prism, version 5.00 for Windows (GraphPad software, San Diego, CA, USA). Continuous data are presented as medians and interquartile ranges unless stated otherwise.

\section{Results}

During the study period, 83 women underwent cholecystectomy. Conventional four-port laparoscopic cholecystectomy was chosen for 33 of the patients and open cholecystectomy for 1 patient, and these patients were therefore not included in the study. A total of 49 patients underwent tvCCE. Of these 49 patients, 15 were excluded from the study because they declined to participate $(n=2)$ or the surgeon was not familiar with IOC in tvCCE at the time of surgery $(n=6)$. For 7 of these 15 cases, the dedicated research fellow was not available, and the patients were therefore not enrolled in the study. Consequently, 33 patients were included in the study and 49 were excluded (Fig. 3).

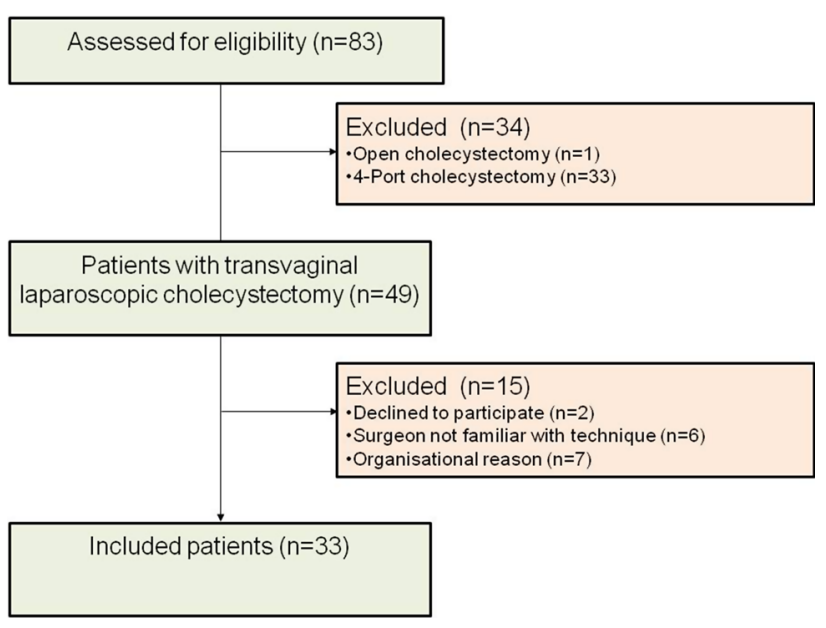

Fig. 3 Patient flow diagram

The median age of the included patients was 45 years (interquartile range [IQR], 34-60 years), and the median BMI was $24 \mathrm{~kg} / \mathrm{m}^{2}$ (IQR, $23-28 \mathrm{~kg} / \mathrm{m}^{2}$ ). The indication for tvCCE was elective for 28 patients with symptomatic cholecystolithiasis $(n=27)$ or a history of acute biliary pancreatitis $(n=1)$ and emergent for five patients with acute cholecystitis. Of the 33 patients, 8 had a history of lower abdominal surgery.

Table 1 depicts the baseline characteristics, indication for surgery, and previous abdominal surgery for the included and excluded patients. The patients excluded from the study were more likely than the included patients to present with acute cholecystitis (41 vs $15 \%$ ), a history of abdominal surgery (43 vs $24 \%$ ), and a history of pancreatitis or choledocholithiasis (16 vs $3 \%$ ). They also tended to be older (median age, 61; IQR, 38-72 years vs median age, 45 years; IQR, 34-60 years).

Preoperative ultrasonography was performed for all the patients. This examination showed stones in the gallbladder of all the patients, as well as a thickened gallbladder wall in the five patients with acute cholecystitis. In no case was dilation of the ductus hepatocholedochus or the intrahepatic biliary tracts noted.

An MRCP obtained for seven patients showed cholecystolithiasis in all seven patients, a thickened gallbladder wall in two patients, and signs of acute pancreatitis and choledocholithiasis in one patient each. In the latter case, an ERCP with papillotomy and successful clearing of the common bile duct (CBD) was performed preoperatively (Table 2).

In 32 cases of tvCCE (97\%), one of which had to be converted to conventional four-port laparoscopic cholecystectomy, an intraoperative cholangiogram was performed successfully. In one patient, the intubation of a very thin cystic duct failed because of a mismatch between the sizes of the cystic duct and catheter. In this case, cholecystectomy 
Table 1 Baseline characteristics of included $(n=33)$ and excluded $(n=49)$ patients

\begin{tabular}{lll}
\hline & $\begin{array}{l}\text { Included } \\
\text { patients } \\
(n=33)\end{array}$ & $\begin{array}{l}\text { Excluded } \\
\text { patients } \\
(n=49)\end{array}$ \\
\hline Age, median (IQR) (years) & $45(34-60)$ & $61(38-72)$ \\
BMI, median (IQR) $\left(\mathrm{kg} / \mathrm{m}^{2}\right)$ & $23(23-28)$ & $25(24-29)$ \\
Symptomatic cholecystolithiasis, $n(\%)$ & $27(82)$ & $21(43)$ \\
Acute cholecystitis, $n(\%)$ & $5(15)$ & $20(41)$ \\
History of pancreatitis, $n(\%)$ & $1(3)$ & $5(10)$ \\
History of choledocholithiasis, $n(\%)$ & $0(0)$ & $3(6)$ \\
Patients with previous open or & $8(24)$ & $21(43)$ \\
laparoscopic surgery, $n(\%)$ & & \\
Number of previous surgical interventions & & \\
Overall number & 10 & 23 \\
$\quad$ Appendectomy & 2 & 19 \\
Adnexectomy & 1 & 1 \\
Cystectomy & 1 & 0 \\
Hysterectomy & 4 & 2 \\
Cesarean section & 2 & 1
\end{tabular}

$I Q R$ interquartile range, $B M I$ body mass index

Table 2 Ultrasonography, MRCP, and ERCP $(n=33) n(\%)$

\begin{tabular}{lc}
\hline Ultrasonography & $33(100)$ \\
Cholecystolithiasis & $30(90)$ \\
With acute cholecystitis & $5(15)$ \\
With sludge & $2(6)$ \\
With contracted gallbladder & $1(3)$ \\
MRCP & $7(21)$ \\
Cholecystolithiasis & $7(21)$ \\
With acute cholecystitis & $2(6)$ \\
With pancreatitis & $1(3)$ \\
With choledocholithiasis & $1(3)$ \\
ERCP & $2(6)$ \\
Preoperative & $1(3)$ \\
Choledocholithiasis & $1(3)$ \\
Postoperative & $1(3)$ \\
Persistent choledocholithiasis & $1(3)$ \\
\hline
\end{tabular}

$M R C P$ magnetic resonance cholangiopancreatography, ERCP endoscopic retrograde cholangiopancreatography

was performed without a cholangiogram, and no complication occurred.The median duration of surgery was $56 \mathrm{~min}$ (IQR, 45-65 $\mathrm{min}$ ), and the median cholangiography time was 6 min (IQR, 4-7 min).

Two types of cholangiography catheters were used. The median duration of cholangiography was $6 \mathrm{~min}$ (IQR, $5-6 \mathrm{~min})$ using the Coeliodrain) $(n=13)$ and $5 \mathrm{~min}$ (IQR, 3-7 min) using the Kendall Argyle (Tyco Healthcare)
Table 3 Reasons for additional ports as well as their numbers, locations, and types: 14 of $33(42 \%) n(\%)$

\begin{tabular}{ll}
\hline Reasons for additional ports & \\
\hline Intraabdominal adhesions & $4(12)$ \\
Obesity & $2(6)$ \\
Inability to achieve a critical view of safety & $5(15)$ \\
Need for revision of choledochus & $3(9)$ \\
Numbers, locations, and types of additional ports & \\
One 5-mm port, right upper quadrant & $7(21)$ \\
Two 5-mm ports, right and left upper quadrants & $3(9)$ \\
One 12-mm port, subxyphoidal & $3(9)$ \\
One 2-mm port, right upper quadrant & $1(3)$ \\
\hline
\end{tabular}

$(n=19)$. The quality of the obtained intraoperative cholangiograms was good in all cases, showing clearly the anatomy of the biliary tract, the intrahepatic biliary system, and the drainage of contrast in the duodenum.

Additional abdominal ports were required in $14(42 \%)$ of the 33 patients for safe completion of the procedure. In none of the patients was the need for additional ports related to the diagnostic cholangiography itself. The reasons for the introduction additional cannulas as well as their numbers, locations, and types are given in Table 3 . One conversion ( $3 \%$ ) to conventional 4-port laparoscopic cholecystectomy was performed because of severe adhesions with the lesser pelvis precluding a transvaginal approach. The conventional laparoscopic cholecystectomy was uneventful in this case. No conversion to open surgery was necessary.

In three patients $(10 \%)$, CBD stones were detected incidentally during cholangiography, and a laparoscopic bile duct revision was performed. Only one of these three patients had undergone a preoperative MRCP, which showed no prepapillary concrement. In none of these patients did preoperative ultrasonography show congestion of the CBD or the intrahepatic biliary system (Fig. 4). For laparoscopic bile duct revision in all three patients, one additional 12-mm port (Versastep; Covidien, Norwalk, CT, USA) was placed in the subxyphoidal position.

The transcystic bile duct revision was performed using a Fogarty catheter in one patient and a Fogarty catheter in combination with a Dormia basket in two patients. This procedure was successful in two cases. After bile duct revision, the gallbladder was removed transvaginally. In one patient, a prepapillar stone could not be removed by intraoperative bile duct revision and was retrieved by a postoperative ERCP.

In two patients, additional laparoscopic procedures during the same surgery were performed successfully without additional trocars. The one patient had an appendectomy (suspicion of neurogenic appendicitis), and the 
Fig. 4 Overview of results from pre-, intra-, and postoperative assessment of treatment for choledocholithiasis

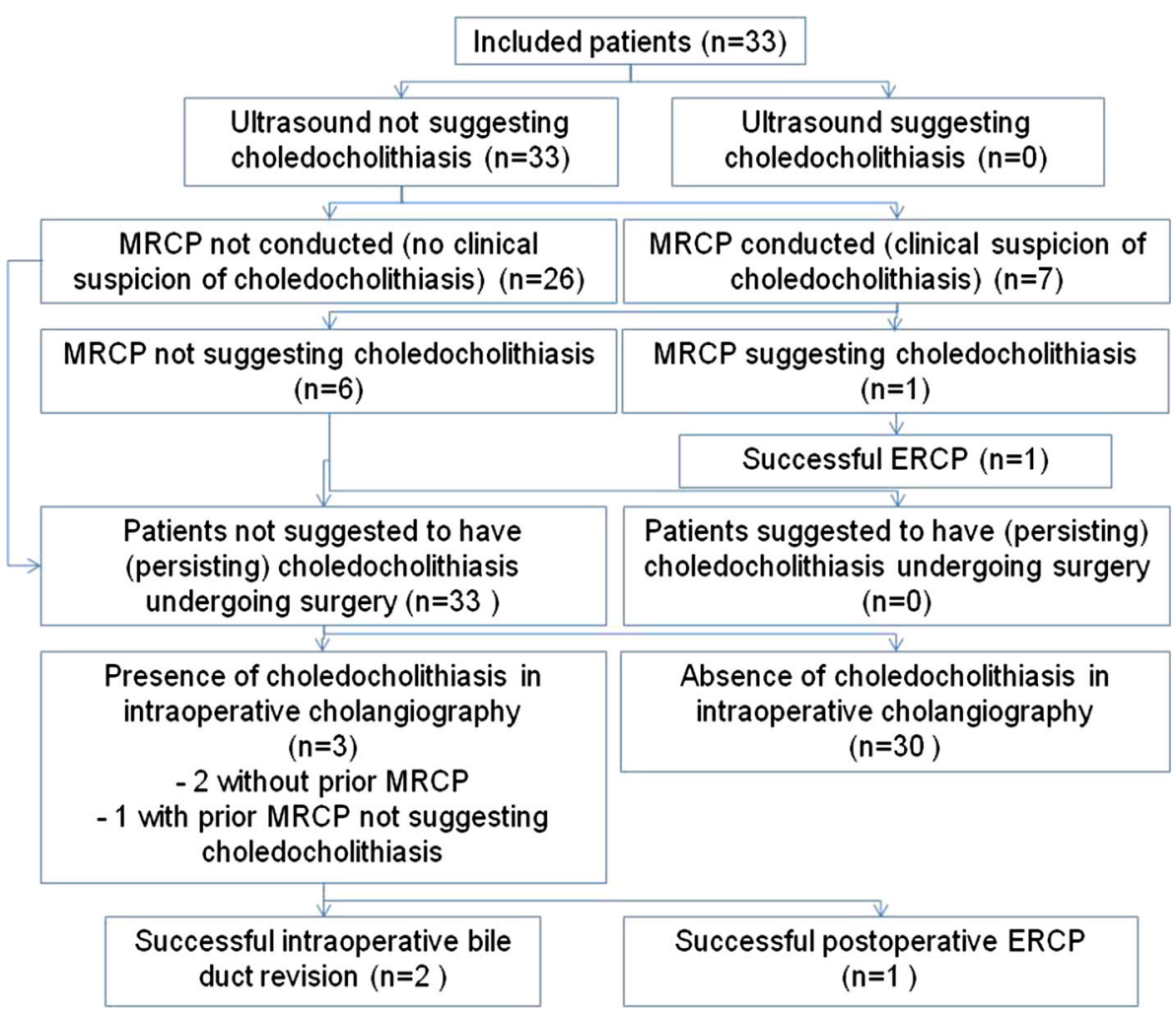

Table 4 Intra- and postoperative complications $(n=33) n(\%)$

\begin{tabular}{ll}
\hline Intraoperative complications & $3(9)$ \\
Perforation of the gallbladder & $2(6)$ \\
Failure of intraoperative cholangiography & $1(3)$ \\
Postoperative surgical complications & $1(3)$ \\
Persisting right upper quadrant pain (grade 1) & $1(3)$
\end{tabular}

other patient had puncture of an ovarian cyst. The operations were performed by three laparoscopically experienced, board-certified staff surgeons.

Among the 33 operations, three intraoperative complications $(10 \%)$ and one postoperative complication (3\%) occurred (Table 4). The median postoperative hospital stay was 2 days (range, 2-3 days). One patient had a hospital stay of 11 days due to a newly diagnosed fracture of vertebral body L1 and several rib fractures after a fall 2 weeks before the operation and prolonged back pain after surgery.

The gynecologic control visit showed pathologic findings in four patients (12\%) including one bacterial vaginosis, one transposition of an intrauterine pessar, and two cases of dysplastic cells in the cervical smear test.

\section{Discussion}

This is the first prospective case series to demonstrate the feasibility and safety of IOC in tvCCE. Cholangiography was successful in $97 \%$ of the cases without conversion. The duration of cholangiography was short, with a median of $6 \mathrm{~min}$. Except for the impossibility of intubating a very thin cystic duct in one patient, no specific problems or complications related to cholangiography were recorded.

The tvCCE technique has become a routine procedure in a few specialized laparoscopic departments. But among general and visceral surgeons, mistrust of this new technique persists. Nevertheless, many suspected hurdles have been eliminated by good evidence.

The feasibility of tvCCE has been shown in well-performed prospective case series and cohort studies [1-6], and suspected gynecologic complications such as dyspareunia or impaired sexual function have been invalidated. In one study, no evidence of sexual impairment, as assessed by a validated sexual functioning score, was found 1 year after transvaginal natural orifice translumenal endoscopic surgery (NOTES), and patient satisfaction with transvaginal surgery was as high as $96 \%$ [23].

Intraoperative cholangiography, as an adjunct to verifying the anatomy of the biliary system in case of doubt, plays an important role in preventing CBD injury. Furthermore, it helps in the early identification of such injury, enabling its best management.

Whether the routine use of IOC reduces the rate of CBD injury or not still is a matter of debate $[13,15,17,18]$, and most surgeons perform IOC selectively to verify the biliary anatomy in unclear situations or in cases of suspected 
injury. Furthermore, it is used if CBD stones are suspected [14].

The current study demonstrated that the potential need for IOC is not a contraindication for tvCCE. In this study, two types of cholangiography catheters were used. After 13 procedures, the Coeliodrain catheter was replaced by the Argyle umbilical vessel catheter (Covidien plc, Dublin, Ireland) because intubation of the cystic duct was thought to be easier with the smoother tip of the Argyle catheter. The Argyle catheter is made of polyurethane and originally was designed for venipuncture of umbilical veins in premature infants. However, the success rate and the median time of cholangiography did not differ between the two catheters.

The feasibility of CBD revision during tvCCE has not been evaluated previously. In this study, it was successful for two of three patients with intraoperative findings of preoperative occult $\mathrm{CBC}$ stones. In all three patients, who underwent $\mathrm{CBD}$ revision, one additional $12-\mathrm{mm}$ port was introduced in the subxyphoidal position. The transvaginal hybrid-NOTES technique offers the possibility introducing several additional ports if needed, enabling more advanced laparoscopic procedures such as a CBD revision.

One or two additional 2- to $12-\mathrm{mm}$ ports were used in $42 \%$ of the patients. The main reason for the additional cannula was to improve retraction of the gallbladder for safe exposure of Calot's triangle. In some cases, exposure of Calot's triangle with only a single retaining grasper may be difficult and might not allow establishment of an adequate critical view of safety. In such situations, surgeons should not hesitate to use additional small ports to improve exposure of Calot's triangle. In our opinion, the main advantage of tvCCE is its avoidance of a minilaparotomy to remove the gallbladder because virtually no pain is felt at the colpotomy site.

The results of this study may be limited to a certain patient group due to selection bias because both the surgeons and the patients were free to select either a transvaginal or a four-port laparoscopic cholecystectomy. Furthermore, there was an organizational bias because 15 patients undergoing tvCCE were not included in the study due to inavailability of a surgeon experienced with IOC in tvCCE (namely, in August 2012). Nevertheless, at the termination of this study, all the staff surgeons in our department had been well trained to perform IOC in tvCCE. Furthermore, the dedicated research fellow was absent in October 2012. Except for these periods, the inclusion of tvCCE patients in the study was consecutive.

There was an imbalance in baseline characteristics between the patients excluded from the study and those included. The included patients potentially represented the less complex cases, which may limit generalizability to such cases. Nevertheless, the inclusion of some acute cholecystitis cases and the successful management of CBD stones with the addition of one port in two patients suggest that more complex procedures may be addressed with a transvaginal hybrid access.

In conclusion, by demonstrating the feasibility and safe performance of IOC in tvCCE, this study has removed a further hurdle in the routine use of this promising technique. Moreover, the feasibility of $\mathrm{CBD}$ revision in tvCCE has been demonstrated.

Disclosures Önder Ögredici, Georg R. Linke, Sebastian Lamm, Rachel Rosenthal, Andreas Zerz, and Daniel C. Steinemann have no conflicts of interest or financial ties to disclose.

\section{References}

1. Zornig C, Emmermann A, von Waldenfels HA, Mofid H (2007) Laparoscopic cholecystectomy without visible scar: combined transvaginal and transumbilical approach. Endoscopy 39:913-915

2. Zornig C, Mofid H, Emmermann A, Alm M, von Waldenfels HA, Felixmüller C (2008) Scarless cholecystectomy with combined transvaginal and transumbilical approach in a series of 20 patients. Surg Endosc 22:1427-1429

3. Zornig C, Mofid M, Emmermann A, Alm M, Waldenfels H-A, Freimüller C (2009) NOTES-Cholecystektomie ohne Narben. Chirurg 80:364-369

4. Linke GR, Tarantino I, Hoetzel R, Warschkow R, Lange J, Lachat R, Zerz A (2010) Transvaginal rigid-hybrid NOTES cholecystectomy: evaluation in routine clinical practice. Surg Endosc 42:571-575

5. Cardoso Ramos A, Muratami A, Galvoneto M, Santana Galvao M, Souza Silva AC, Gonzalo Canseco E, Moyes Y (2008) NOTES transvaginal video-assisted cholecystectomy: first series. Endoscopy 40:572-575

6. Zornig C, Siemssen L, Emmermann A, Alm M, von Waldenfels HA, Felixmüller C, Mofid H (2011) NOTES cholecystectomy: matched-pair analysis comparing the transvaginal hybrid and conventional laparoscopic techniques in a series of 216 patients. Surg Endosc 25:1822-1826

7. Van den Boezem PB, Kruyt FM, Stommel MW, Samlal RA, Sietses C (2011) Cholecystectomy without visible scars: the transvaginal method. Ned Tijdschr Geneeskd 155:A3617

8. Santos BF, Teitelbaum EN, Arafat FO, Milad MP, Soper NJ, Hungness ES (2012) Comparison of short-term outcomes between transvaginal hybrid NOTES cholecystectomy and laparoscopic cholecystectomy. Surg Endosc 26:3058-3066

9. Solomon D, Shariff AH, Silasi DA, Duffy AJ, Bell RL, Roberts KE (2012) Transvaginal cholecystectomy versus single-incision laparoscopic cholecystectomy versus four-port laparoscopic cholecystectomy: a prospective cohort study. Surg Endosc 26:2823-2827

10. Linke GR, Tarantino I, Bruderer T, Celeiro J, Warschkow R, Tarr PE, Müller-Stich BP, Zerz A (2012) Transvaginal access for NOTES: a cohort study of microbiological colonization and contamination. Endoscopy 44:684-689

11. Agarwal BB, Mahajan KC (2010) Laparoscopic biliary tract injury prevention: zero tolerance, error free performance. Surg Endosc 24:728-729

12. Kum CK, Eypasch E, Lefering R, Paul A, Neugebauer E, Troidl H (1996) Laparoscopic cholecystectomy for acute cholecystitis: Is it really safe? World J Surg 20:43-48 
13. Schmitz SF, Krähenbühl S, Krähenbühl L (2010) Bile duct injury and use of cholangiography during laparoscopic cholecystectomy. Br J Surg 98:391-396

14. Connor S, Garden OJ (2006) Bile duct injury in the era of laparoscopic cholecystectomy. Br J Surg 93:158-168

15. Ford JA, Soop M, Du J, Loveday BP, Rodgers M (2012) Systematic review of cholangiography in cholecystectomy. Br J Surg 99:160-167

16. Nuzzo G, Giuliante F, Giovanni I, Ardito F, D'Acapito F, Vellone M, Murazio M, Capelli G (2005) Bile duct injury during laparoscopic cholecystectomy: results of an Italian national survey on 56,591 cholecystectomies. Arch Surg 140:986-992

17. Davidoff AM, Pappas TN, Murray EA, Hilleren DJ, Johnson RD, Baker ME, Newman GE, Cotton PB, Meyers WC (1992) Mechanisms of major bile duct injury during laparoscopic cholecystectomy. Ann Surg 215:196-202

18. Tylor OM, Sedman PC, Jones BM, Royston CM, Arulampalam T, Wellwood J (1997) Laparoscopic cholecystectomy without intraoperative cholangiogram: 2,038 cases over a 5-year period in two district general hospitals. Ann R Coll Surg Engl 79:376-380
19. Dindo D, Demartines N, Clavien PA (2004) Classification of surgical complications: a new proposal with evaluation in a cohort of 6,336 patients and results of a survey. Ann Surg 240:205-213

20. Trichat S (2003) Three-port versus four-port laparoscopic cholecystectomy. Surg Endosc 17:1434-1436

21. Strasberg SM, Hertl M, Soper NJ (1995) An analysis of the problem of biliary injury during laparoscopic cholecystectomy. J Am Coll Surg 180:101-125

22. Vettoretto N, Saronni C, Harbi A, Balestra L, Taglietti L, Giovanetti M (2011) Critical view of safety during laparoscopic cholecystectomy. J Laparoendosc Surg 15:322-325

23. Linke GR, Luz S, Janczak J, Zerz A, Schmied BM, Siercks I, Warschkow R, Beutner U, Tarantino I (2013) Evaluation of sexual function in sexually active women 1 year after transvaginal NOTES: a prospective cohort study of 106 patients. Langenbecks Arch Surg 398:139-145 